\title{
Simple modelling approach using Modelica for microbial electrosynthesis
}

\author{
Gamunu Samarakoon ${ }^{1}$ Dietmar Winkler ${ }^{2} \quad$ Vasan Sivalingam $^{1} \quad$ Carlos Dinamarca $^{1} \quad$ Rune Bakke $^{1}$ \\ ${ }^{1}$ Department of Process, Energy and Environmental Technology, ${ }^{2}$ Department of Electrical Engineering, Information \\ Technology and Cybernetics, University of South-Eastern Norway, Porsgrunn 3918, Norway, \\ \{gamunu.arachchige, dietmar.winkler, vasan.sivalingam, \\ carlos.dinamarca, rune.bakke@usn.no\}
}

\begin{abstract}
This study intends to develop a simple mathematical model that contributes to the integration of Microbial Electrosynthesis (MES) in AD to reduce $\mathrm{CO}_{2}$ to $\mathrm{CH}_{4}$. Open-source modelling language Modelica was used to build the model. The MES internal resistances are important parameters for the model and an Electrochemical Impedance Spectroscopy (EIS) experiment was employed to estimate the resistances and distinguish the contribution from each resistance element. The model preliminary simulations show that it is possible to determine the voltage required to keep the potential difference across the cathode biofilm within optimal conditions. The system is sensitive to effects of biofilm development on electron transfer at both electrodes, which implies effects on the electrons from anode to cathode (i.e. electric current). The model will be a useful tool for extrapolating experimental results and to enhance our understanding of MES.
\end{abstract}

Keywords: microbial electrosynthesis, bio-methane, $\mathrm{CO}_{2}$ reduction, Modelica

\section{Introduction}

Microbial electrosynthesis is a novel technology for chemical synthesis of desired product through chemical reaction catalysed by microorganisms and powered by electric energy (Rabaey and Rozendal, 2010). MES occurs in microbial electrolysis cell. The working electrode (WE) of the microbial electrolysis cell is in general the cathode at which the reduction half reaction is controlled by the potential to achieve the desired product.

Recently, MES for biogas upgrading (Nelabhotla and Dinamarca, 2018) by $\mathrm{CO}_{2}$ (carbon dioxide) reduction to $\mathrm{CH}_{4}$ (methane) has received attention. A typical biogas reactor (anaerobic digester; AD) produces biogas containing $50-70 \% \mathrm{CH}_{4}$ and $30-50 \% \mathrm{CO}_{2}$, implying low calorific value (Angelidaki et al., 2018). Biogas upgrading by MES should preferably use electricity from renewable sources and may thereby also serve as a way of storing renewable surplus electricity as $\mathrm{CH}_{4}$
(Geppert et al., 2016). Other valuable chemical products from $\mathrm{CO}_{2}$ may also be obtained by adjusting the MES cathode potential (Schievano et al., 2019).

The conversion of $\mathrm{CO}_{2}$ to $\mathrm{CH}_{4}$ occurs at the cathode through direct electron transfer (Table 1, eq. (1)) or indirectly via production of intermediates. The conversion of $\mathrm{CO}_{2}$ to $\mathrm{CH}_{4}$ with intermediate production of hydrogen $\left(\mathrm{H}_{2}\right)$ follows two steps: protons reduction to $\mathrm{H}_{2}$ (Table 1, eq. (2.1)) and then the produced $\mathrm{H}_{2}$ is used as electron donor for biological $\mathrm{CO}_{2}$ reduction to $\mathrm{CH}_{4}$ (Table 1, eq. (2.2)). $\mathrm{CH}_{4}$ production is also possible from acetate $\left(\mathrm{CH}_{3} \mathrm{COO}^{-}\right)$produced bioelectrochemically via $\mathrm{CO}_{2}$ reduction.

Table 1. Chemical reactions at cathode with standard potential (S.P.) [4] vs. Normal Hydrogen Electrode (NHE)

\begin{tabular}{|l|rr|}
\hline Reactions at cathode & S.P. [V] & \\
\hline $\mathrm{CO}_{2}+8 \mathrm{H}^{+}+8 e^{-} \rightarrow \mathrm{CH}_{4}+2 \mathrm{H}_{2} \mathrm{O}$ & -0.24 & $(1)$ \\
\hline $8 \mathrm{H}^{+}+8 e^{-} \rightarrow 4 \mathrm{H}_{2}$ & -0.41 & $(2.1)$ \\
$\mathrm{CO}_{2}+4 \mathrm{H}_{2} \rightarrow \mathrm{CH}_{4}+2 \mathrm{H}_{2} \mathrm{O}$ & & (2.2) \\
\hline
\end{tabular}

The standard potential of $\mathrm{CH}_{4}$ production via direct electron transfer (- $0.24 \mathrm{~V}$ vs. NHE) is lower than that of an indirect electron transfer $(-0.41 \mathrm{~V}$ vs. NHE) (Table 1), implying that direct electron transfer is the more energy efficient path. The cathode potential also influences current (electron flow) and hence the $\mathrm{CH}_{4}$ production rate (Geppert et al., 2016) and is the key parameter that determines mechanism of electron transfer.

The protons $\left(\mathrm{H}^{+}\right)$and electrons $(e)$ needed for the reduction reaction at the cathode are generated at the anode, such as by oxidizing water eq. (3) or easily degradable short-chain volatile fatty acid (VFA) such as acetate eq. (4). Oxidation of these components may have some downsides. Thus, other possible oxidation reactions, e.g., ammonium oxidation (Sivalingam et al., 2020), may be relevant.

$$
2 \mathrm{H}_{2} \mathrm{O} \rightarrow 4 \mathrm{H}^{+}+\mathrm{O}_{2}+4 e^{-} \quad 0.82 \mathrm{~V} \text { vs. NHE }
$$




$$
\mathrm{CH}_{3} \mathrm{COO}^{-} \mathrm{H}^{+}+2 \mathrm{H}_{2} \mathrm{O} \rightarrow 2 \mathrm{CO}_{2}+8 \mathrm{H}^{+}+8 e^{-}
$$

$-0.28 \mathrm{~V}$ vs. NHE

The minimum voltage required to bring about electrolysis can be estimated from the standard reduction potentials of desired reaction and is called thermodynamic cell voltage eq. (5).

$$
E_{\text {cell }}^{0}=E_{\text {cath }}^{0}-E_{\text {anod }}^{0}
$$

However, the applied cell voltage consists of not only the thermodynamically calculated cell voltage, but also internal energy losses or over-potentials. These internal losses originate from three sources; activation overpotential ( $\eta_{\text {act }}$, related to the rates of electrode reaction), concentration over-potential ( $\eta_{\text {conc }}$, related to mass transfer limitations of chemical species transported to or from the electrode), ohmic over-potential ( $\eta_{\mathrm{ohm}}$,related to the resistance to the flow of ions in the electrolyte and to the flow of electrons through the electrode material) (Picioreanu et al., 2007). The activation and concentration over-potentials incur separately both at cathode and anode, see eq. (6).

$$
\begin{aligned}
& V_{\text {cell }}=\left(E_{\text {cath }}^{0}-\eta_{C, a c t}-\eta_{C, \text { con }}\right)-\left(E_{\text {anod }}^{0}-\eta_{A, a c t}-\right. \\
& \left.\eta_{A, \text { conc }}\right)-\eta_{\text {ohm }}
\end{aligned}
$$

\subsection{Electrochemical Impedance Spectroscopy and Equivalent Electrical Circuit}

The electrochemical balance eq. (6) expressed as the potential drops at each electrode are influenced by the resistances to the bioelectrochemical reactions and electrochemical processes in MES. These resistances can be converted into an Equivalent Electrical Circuit (EEC) (Jiya et al., 2018). Figure 1 shows a typical EEC of an electrode, where $\mathrm{C}$ is the capacitance of the electrical double layer (between the electrode and the electrolyte solution), $R_{p}$ represents charge transfer resistance originating from MES reactions (related to the activation energy of the electrodes) and $\mathrm{R}$ represents resistance by the electrolyte solution. The electrochemical impedance spectroscopy (EIS) has been used to characterize electrochemical system by means of EECs (Wagner, 2002).

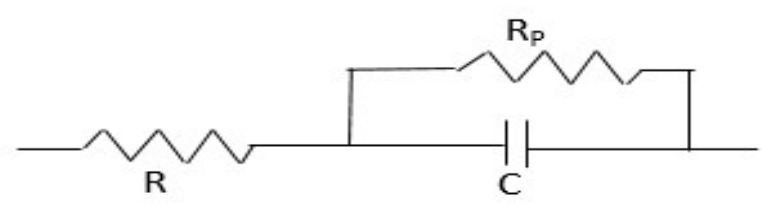

Figure 1: Classical equivalent circuit of a double layer capacitor showing its three basic characteristics: The internal resistance (R), its Capacitance (C), and selfdischarge resistance (Rp) (Jiya et al., 2018).

EIS characterizes the response of an electrical circuit to alternative current (AC) or voltage. For a certain amplitude and frequency of applied AC signal, the circuit responds with a particular amplitude of alternating current at the same frequency. This response is quantified as impedance based on Ohm's law $(\mathrm{E}=\mathrm{I} Z$, where $\mathrm{E}$ and $\mathrm{I}$ are $\mathrm{AC}$ voltage and current respectively and $\mathrm{Z}$ is the impedance). The impedance depends on the frequency of the applied signal (voltage) and the time shift (or phase shift) between the input and output signals.

This technique can be applied to electrochemical cells (in this case MES). An AC signal is applied to the cell and the corresponding response (the impedance) depicts the resistance to charge flow. The charge flow depends on the bio electrochemical reactions and processes in the cell. The different frequencies of the applied AC voltage can distinguish the different processes that have different time scales. The lower frequencies are corresponding to the slow processes such as diffusion and slow electrochemical reactions. The higher frequencies are corresponding to the faster processes. At higher frequencies we can approximate the impedance due to the flow of ions in the electrolyte (i.e., the resistance of the electrolyte solution). Then the impedance response is mainly due to the charge and discharge of the double layer (capacitance).

The impedance data is generated in the form of a spectrum typically on a plot called Nyquist plot. The response impedance $\mathrm{Z}$ is composed of a real and imaginary part. The "Nyquist Plot" represents the real part on $\mathrm{X}$-axis and the imaginary part on $\mathrm{Y}$-axis. Bode plot is also another important graph used in EIS analysis where the absolute values of the frequency response (i.e., impedance) and the phase-shift are plotted vs the applied frequency.

The EEC associated with the corresponding spectrum can be constructed with the amplitude of the circuit elements using computer aided tools.

\subsection{Aims}

This study intends to develop a simple mathematical model that contributes to the integration of MES in AD to reduce $\mathrm{CO}_{2}$ to $\mathrm{CH}_{4}$. The experimental work on this is so far limited and will require large efforts to test wide variety of operational conditions, while mathematical modelling can extrapolate on such results and enhance our understanding of MES. In this case, our aim is to develop a simple model to understand the variation in resistances in a MES system as the biofilm grow and in its fully-grown operation. An EIS experiment is employed to estimate the resistances of the electrolyte solution and the charge transfer and distinguish the contribution from each to the MES internal resistance. The Open-source Modelling language Modelica (Modelica Association, 2017) is used to build the simple model. Typical applications of the models as such also include design of power management systems from lab.- to full-scale reactor setups, and the biofilm 
resistance values are important to understand the kinetic behaviours of bio-electrochemical reactions.

\section{Materials and methods}

\subsection{MES reactor and operation}

The present MES experiments were performed in a $100 \mathrm{~mL}$ Borosilicate glass bottle with a 3-ports Teflon screw cap. The reactor was operated in a semicontinuous mode. A carbon nanotube composite (made in-house, area $\sim 1 \mathrm{~cm}^{2}$ ) was used as the cathode. A Graphite rod (L: $152 \mathrm{~mm} \times$ D: $6.15 \mathrm{~mm}$; Alfa Aesar, Thermo Fisher GmbH, Karlsruhe, Germany) was used as the anode. An Ag/ $\mathrm{AgCl}$ electrode (+0.209 V vs. SHE; $3 \mathrm{M} \mathrm{NaCl}$, QVMF2052, ProSense, BB Oosterhout, The Netherlands) was used as the reference electrode. The electrodes were connected with titanium wire. The voltage between cathode and reference was kept constant at $-0.42 \mathrm{~V}$ vs SHE by Potentiostat (Interface 1000B, Gamry instruments, Pennsylvania, USA) with the intention of synthesising $\mathrm{CH}_{4}$.

The reactor was operated at $35^{\circ} \mathrm{C}$ and 10 days HRT with a fully growth biofilm condition. A synthetic feed prepared according to Kenarsari et al. (2020) at a flow rate of $10 \mathrm{~mL} /$ day was used. Anaerobic digester sludge from the local municipal wastewater treatment plant (Knarrdalstrand, Porsgrunn, Norway) was used as the inoculum.

\subsection{EIS experiment}

The current EIS experiment results were generated by running EIS in a cell with a fully-grown biofilm cathode. This experiment work was type of "lesson-learned" and our first attempt was to estimate the impedance associated with the cathode (i.e., WE). We relied on the EIS instrument capacity available in our lab at the time of the study (We intent to improve the capacity and expand our knowledge targeting a pilot scale MES). The EIS experiment was performed by using a potentiostat (Interface 1000B, Gamry instruments, Pennsylvania, USA). The initial frequency was set to $20 \mathrm{kHz}$, which is the maximum limit of interface $1000 \mathrm{~B}$ and stepped down to $0.2 \mathrm{~Hz}$ through 10 points/decade ratio. The impedance data is generated in the form of a spectrum on Nyquist plot and the Bode plot. The two plots were used to estimate the electrolyte solution resistance. On the Nyquist plot, the intercept of the spectrum with the $\mathrm{X}$-axis (real part of the impedance) in the highfrequency range gives the electrolyte resistance. The impedance in the high frequency range and zero phaseshift was confirmed using Bode plot.

\subsection{Model development}

The equivalent electrical circuit as given in Figure 1 can be further simplified depending on the application.
Though the biofilm can be represented as an impedance consisting mainly of both resistance and capacitance, in a MES application we will be purely dealing with direct current flows. This means that the capacitance does not have any significant influence on the electrical behaviour of the reactor. Furthermore, we assumed that the resistance of the anode (connected to the counterelectrode, (CE) of the potentiostat) side is negligible compared to the resistance of the solution and biofilm (for this stage of model development). The electrolyte solution itself can be represented by a resistance that also does not change over time since the ionic strength of the solution will stay almost the same.

Figure 2 shows the developed model consisting of an ideal regulated potentiostat voltage source, including sub-models for the development of electrode biofilms and solution properties. The model was build using the open-source Modelling language Modelica (Modelica Association, 2017).

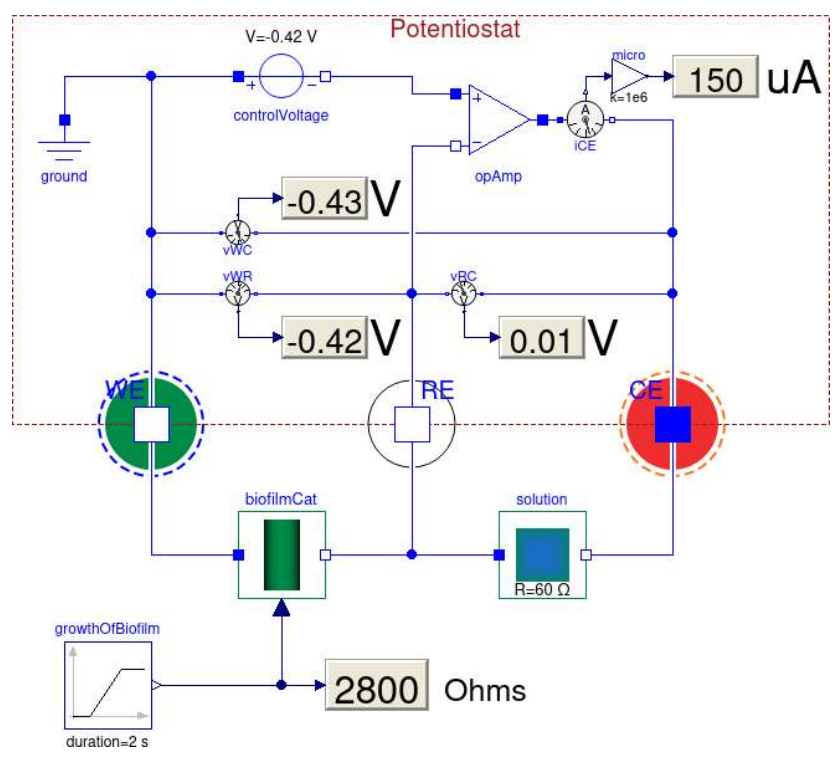

Figure 2: Graphical presentation of potentiostat controlled MES for $\mathrm{CO}_{2}$ reduction to $\mathrm{CH}_{4}$ model implemented in Modelica.

\section{Result and discussion}

Based on our EIS experiment, we could estimate the electrolyte solution resistance at the cathode side as $60 \Omega$ by observing the Nyquist plot and the Bode plot. Our estimation is based on the frequency response of the highest frequency we could reach with our EIS instrument capacity and the frequency response at zero phase shift. The highest frequency was $20 \mathrm{kHz}$. 

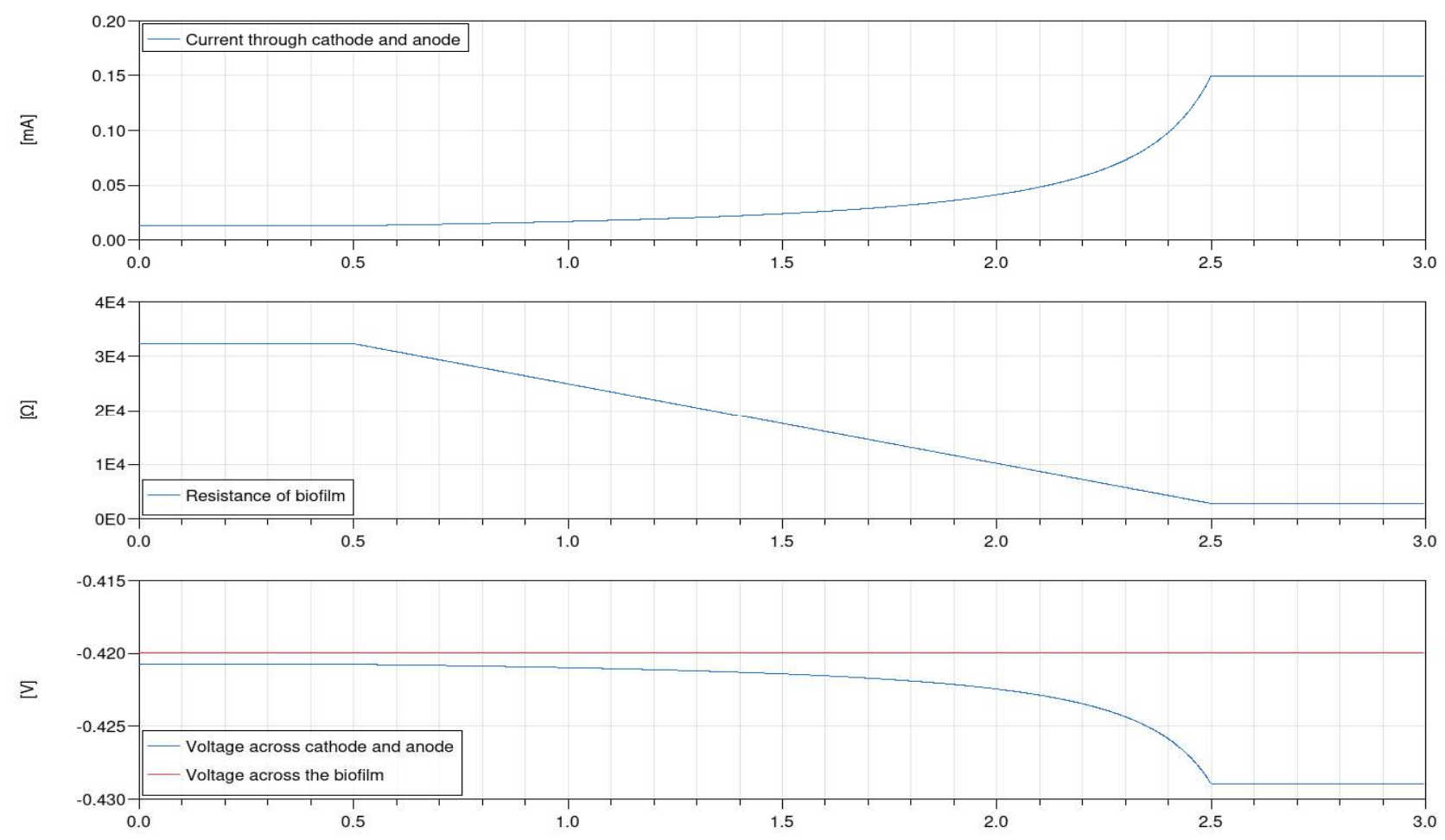

Figure 3: Simulation results for biofilm growth over time in seconds (in reality the growth happened over 21 days).

\subsection{Simulation result}

The model presented in Figure 2 is used to verify if the voltage level between anode and cathode does not exceed a certain set value (above which undesirable oxidation reactions can occur at the anode or CE) whilst still keeping the voltage across the biofilm at the optimal value of V_biofilm $=-0.42 \mathrm{~V}$ at the cathode (WE) for $\mathrm{CH}_{4}$ production.

The resistance between anode and solution is neglected for this stage of model development. The resistance of the solution (R_solution) itself has been experimentally determined to be $=60 \Omega$. Also, from measurements obtained from MES experiments, we could find that at the beginning of the biofilm growth (i.e., abiotic condition), the current flow was $13 \mu \mathrm{A}$ and after the growth period of 21 days the current went up to $150 \mu \mathrm{A}$. Those measurement were taken whilst the potential across the biofilm was always kept at a constant, (i.e. V_biofilm $=-0.42 \mathrm{~V}$ ).

According to Ohm's law this translates to a biofilm resistance of about $R_{-}$biofilm $=32 \mathrm{k} \Omega$ at the beginning of the growth period and $3 \mathrm{k} \Omega$ once the biofilm was fully established.

These values were then used in the simulation model in order to determine how high the voltage potential between anode and cathode will get when the voltage drop across the biofilm is kept constant whilst the resistance of the biofilm itself is getting smaller as it is growing.

Figure 3 is showing the simulation results for a change of resistance of the biofilm over time. The maximum voltage drop in this case does not exceed $\mathrm{V}$ biofilm $\leq 0.43 \mathrm{~V}$. So, the model simulations show that it is possible to determine the voltage required to keep the potential difference across the cathode biofilm within optimal conditions. The system is sensitive to effects of biofilm development on electron transfer at both electrodes, which implies effects on the electric current flow in the cell. This is useful for experimental design.

\section{Conclusions and future development}

- A functional mathematical model for the integration of MES in $\mathrm{AD}$ to reduce $\mathrm{CO}_{2}$ to $\mathrm{CH}_{4}$ is developed in Modelica. It is useful in extrapolating experimental results and to enhance our understanding of MES.

- The cathode resistance was reduced by a factor ten when biofilm was established, compared to the clean electrode. This implies an efficient electron transfer and utilization in the cathode where biofilm develops to reduce $\mathrm{CO}_{2}$ to $\mathrm{CH}_{4}$

- The simulations show that it is possible to determine the voltage required to keep the potential difference across the cathode biofilm within optimal conditions (e.g. V_biofilm $\leq$ $0.43 \mathrm{~V}$ for $\mathrm{CO}_{2}$ to $\mathrm{CH}_{4}$ reduction).

\subsection{Further development}

We expect to modify this model to better understand impedance variation in the MES system from the abiotic 
condition (without biofilm), to the fully-grown biofilm operation (i.e., steady state). Hence, the model can predict the cell voltage (across the WE and CE), whilst keeping the optimum potential at the biocathode (or a WE) at different stage of MES operation. EIS experiments will also be carried out accordingly.

\section{References}

Irini Angelidaki, Laura Treu, Panagiotis Tsapekos, Gang Luo, Stefano Campanaro, Henrik Wenzel, and Panagiotis G. Kougias. Biogas upgrading and utilization: Current status and perspectives. Biotechnology Advances, 36(2): 452-466, 2018.

doi:https://doi.org/10.1016/j.biotechadv.2018.01.011.

Florian Geppert, Dandan Liu, Mieke van Eerten-Jansen, Eckhard Weidner, Cees Buisman, and Annemiek ter Heijne. Bioelectrochemical Power-to-Gas: State of the Art and Future Perspectives. Trends in Biotechnology, 34(11): 879894, 2016. doi:https://doi.org/10.1016/j.tibtech.2016.08.010.

Immanuel N. Jiya, Nicoloy Gurusinghe, and Rupert Gouws. Electrical Circuit Modelling of Double Layer Capacitors for Power Electronics and Energy Storage Applications: A Review. Electronics, 7(11): 268, 2018.

Zahra Nikbakht Kenarsari, Nirmal Ghimire, Rune Bakke, and Wenche Hennie Bergland. (2020). Thermophilic Anaerobic Digestion Modeling of Lignocellulosic Hot Water Extract using ADM1. Paper presented at the Proceedings of The 60th SIMS Conference on Simulation and Modelling SIMS 2019, August 12-16, Västerås, Sweden.

Modelica_Association. Modelica ${ }^{\circledR}-\mathrm{A}$ Unified ObjectOriented Language for Systems Modeling, Language Specification, Version 3.4, April 2017. Online: https://www.modelica.org/documents, 2017.

A. B. T. Nelabhotla and C. Dinamarca. Electrochemically mediated $\mathrm{CO}_{2}$ reduction for bio-methane production: a review. Reviews in Environmental Science and Bio/Technology, $\quad$ 17(3): $\quad$ 531-551, 2018. doi:10.1007/s11157-018-9470-5.

Anirudh Bhanu Teja Nelabhotla and Carlos Dinamarca. Bioelectrochemical $\mathrm{CO}_{2}$ Reduction to Methane: MES Integration in Biogas Production Processes. Applied Sciences, 9(6): 1056, 2019.

Cristian Picioreanu, Ian M. Head, Krishna P. Katuri, Mark C. M. van Loosdrecht, and Keith Scott. A computational model for biofilm-based microbial fuel cells. Water Research, 41(13): 2921-2940, 2007. doi:https://doi.org/10.1016/j.watres.2007.04.009.

Korneel Rabaey and René A. Rozendal. Microbial electrosynthesis — revisiting the electrical route for microbial production. Nature Reviews Microbiology, 8: 706, 2010. doi:10.1038/nrmicro2422.

Andrea Schievano, Deepak Pant, and Sebastia Puig. Editorial: Microbial Synthesis, Gas-Fermentation and Bioelectroconversion of $\mathrm{CO}_{2}$ and Other Gaseous Streams. Frontiers in Energy Research, 7(110), 2019. doi:10.3389/fenrg.2019.00110.

Vasan Sivalingam, Carlos Dinamarca, Gamunu Samarakoon, Dietmar Winkler, and Rune Bakke. Ammonium as a
Carbon-Free Electron and Proton Source in Microbial Electrosynthesis Processes. Sustainability, 12(8): 3081, 2020.

N. Wagner. Characterization of membrane electrode assemblies in polymer electrolyte fuel cells using a.c. impedance spectroscopy. Journal of Applied Electrochemistry, 32(8): $\quad 859-863, \quad 2002$. doi:https://doi.org/10.1023/A:1020551609230. 